ing purposes developed from anthracite by-products by the multiple fellowship of the Anthracite Industries Incorporated. The multiple fellowship on heat insulation and proofing has assisted in the development of an efficient insulating material suitable for use for temperatures up to and including $800^{\circ} \mathrm{C}$ The research programme of the multiple fellowship on plastic metals has resulted in the construction of a large commercial sponge-iron plant. Other fellowships have led to improvement in the manufacture of grey cast iron and steel, while a further fellowship has been devoted to the refining of chromium. Steady progress has been made in the utilization of waste pickle liquor under a multiple fellowship of the American Iron and Steel Institute. Five potential processes for the recovery of materials from this industrial waste have been investigated, including the recovery of free sulphuric acid and ferrous sulphate, the manufacture of iron carbonate and ammonium sulphate, the manufacture of magnesium from low-grade ores by heating with waste pickle liquor and the preparation of sodium sulphate and ferric chloride. Other fellowship researches have related to cast iron enamelling, hollow-ware enamelling materials, flat glass and waste grinding sand from plate glass manufacture. Substantial advances in methods for evaluating the flatness and texture of surfaces has been achieved under the multiple fellowship on plate glass technology.

Under the multiple industrial fellowship on sulphur investigations relating to sulphur cements, reaction of gaseous olefines with sulphur and the use of sulphur as a filtering medium are in progress. The same group has discovered a novel series of accelerators for rubber, as well as prepared further organic derivatives from ammonium thiocyanate. The upgrading of the components of coke-oven light oil, the physical properties of tars and pitches and their improvement, as well as the development of new products from tars, the commercial recovery of cyclopentadiene and the recovery of isobutane and isopentane from natural petroleum and casing-head petroleum have been investigated under other fellowships. The feasibility of installing a butane extraction plant, a product used in the manufacture of butadiene for synthetic rubber, has been demon. strated. Other work in the field of natural gas technology has related to corrosion problems. The multiple fellowship on petroleum has again been very active, its investigations including a study of the chemical constitution of high-melting waxes, research on fractional distillation under diffusion pump vacuum and on the chemistry of petroleum.

The multiple fellowship on organic syntheses established by the Carbide and Carbon Chemical Co. has extended its investigations on the manufacture and application of amines, detergents, lubricants, hydraulic fluids and synthetic resins, and a soluble lubricant for processing woollen fibres has been developed. The fellowship on chemical hygiene has continued its investigations on the physiological effects of new chemical compounds including the 'Tergetol' penetrants and the acute oral toxicity of glycol derivatives. The multiple fellowship on food varieties has perfected a new type of instant baby cereal, and initiated a project which will survey the whole field of fruit flavours; problems of the rapid tenderization of meat have also been investigated.

Cotton investigations have included studies on the fibre, the practicability and methods of adapting virgin cotton to the manufacture of high-grade papers, and fundamental research on the properties and structure of cotton cellulose and the influence of such processes as mercerization. Encouraging progress has been made in research on cotton-seed, and the industrial fellowship on felt has devised methods for imparting to paper, machine felts and other woollen industrial fabrics markedly improved physical characteristics and resistance to chemical injury, and has also devised a process for rendering wool and woollen fabrics non-shrinking and nonfelting. An improved system of wool scouring and scouring control has given an average daily increase in production of more than 75 per cent. A third textile fellowship has led to important advances in processing hosiery yarn, particularly 'Nylon'. Yields and grades of glue have been improved by a fellowship on bone products which has also investigated granular absorbents for use in sugar refining. The fundamental problems of rubber technology are being studied by another fellowship, and 'Raolin', a special chlorinated rubber, developed by a Mellon fellowship research, is being allocated according to Federal Government instructions. Research on polymerizable silicon compounds is being carried on under a further fellowship and certain applications have been proposed which are of potential utility in warfare. Other research is related to the use of plastics in water- and gas-meters and for many other purposes.

\section{HEAT ENERGY FROM RADIO- ACTIVE SOURCES IN THE EARTH}

$T$

HE supremely important subject of the amount of heat derived from radioactivity within the earth is the subject of a recent paper by William $D$. Urry, of the Geophysical Laboratory, Washington ("Heat Energy from Radioactive Sources in the Earth", J. Wash. Acad. Sci., 31, 273-84; July, 1941), who has arrived at some very important con clusions. Older methods of determining the rate of heat production in particular specimens by examining the radon or thoron content were very cumbersome and thus few specimens were examined. Urry has taken a suggestion by the Director of the Laboratory and has examined a method of finding this rate of heat production merely by counting the rate of alpha particle emission in an ionization chamber. He finds that

$$
H=2 \cdot 1 \times 10^{-9} \mathrm{~N},
$$

where $H$ is the heat produced in calories per gram of specimen per year and $N$ is the total number of alpha particles emitted per gram per hour from the specimen. Heat accompanying the disintegration of potassium must be added to this and is equal to $4.3 \times 10^{-6} a$ calories per gram per year, where $a$ is gm. $\mathbf{K}_{2} \mathrm{O}$ per $\mathrm{gm}$. of specimen. This equation is possible since an error of less than 5 per cent is intro. duced by taking a fixed thorium uranium ratio in all previously examined types of rocks (acid to ultrabasic) and meteorites (iron and stony) where this ratio ranges from 0 to 20 .

The author has exanined the question of the variation of this heat production in geological history, and estimates that no significant increase in heat production within the structure of the earth has occurred during the accepted age of the earth $\left(2 \times 10^{\circ}\right.$ years $)$. Further, in the opinion of Urry, hitherto undetected 
radio-elements could contribute an appreciable quantity of heat only before $1.5 \times 10^{9}$ years ago.

The new method should facilitate adequate rapid observation (geothermal problems) of great numbers of rock specimens, and should thus in due course present us with a mass of observational data for the study of the influence of heat derived from radioactive materials on the thermal history of the earth, besides contributing data to assist in the solution of present geotectonic problems.

\section{BEHAVIOUR OF SULPHUR IN RUBBER}

A

CCORDING to G. G. Winspear (Bell Lab. Rec., 20, No. 8, April, 1942) vulcanized rubber may have a wide variety of characteristics depending on the amount of sulphur, the period and temperature of vulcanization, and the other substances compounded with it. Identical rubber compositions, vulcanized for the same time and at the same temperature, do not always develop the same characteristics. One of the unsatisfactory conditions that sometimes appears is spottiness, indicating a lack of homogeneity in the product. Before the sulphur assumes a position in the rubber molecule, it dissolves, and to secure uniform solution the sulphur is first dispersed evenly throughout the rubber mass. When sulphur and rubber are mixed on a mill the sulphur is dispersed throughout the rubber mass but most of it is in particles of measurable size. Although the particles may be small, and perhaps even invisible to the unaided eye, they are still very large compared to the sulphur molecule, so that the mixture is far from being one where all the sulphur is in immediate contact with the rubber molecules, which is the theoretical state required to give a homogeneous solution of sulphur in rubber. Such an intimate mixture results from a diffusion of the sulphur of each particle following the dispersion on the mill.

If rubber including a small percentage of sulphur is thoroughly mixed and then held for a long enough time at a high enough temperature, all the sulphur will go into solution. From its original particles the sulphur will diffuse until the whole mass of rubber is thoroughly permeated, and if the temperature is high enough all the sulphur will be dissolved. Rubber in thin sheets with completely dissolved sulphur is clear ard transparent while if it contains undissolved sulphur crystals it will be cloudy. After a known amount of sulphur has all been dissolved in a sheet of rubber some of the sulphur will begin to crystallize out if the temperature is lowered sufficiently, because the lower the temperature the less sulphur will the rubber dissolve. This precipitation of sulphur will be evidenced by the fact that rubber will take on a cloudy appearance.

The interval between mixing and vulcanization is also important. At the end of the mixing period the rubber is at a fairly high temperature and the amount of sulphur in solution will correspond to this temperature. If the rubber is now set aside it will cool and some of the sulphur will crystallize in fine struc. ture dendritic form. When the rubber is again heated in the vulcanizing process the crystals go back readily into solution. If the rubber is allowed to stand for a more extended period before vulcanization, however, the dendritic crystals gradually change to larger rhombic sulphur crystals, so that when the rubber is vulcanized, the sulphur thus crystallized cannot as readily get back into solution because of the time required for completely dissolving these larger crystals. The formation of these rhombic crystals is undoubtedly the cause of some of the spotty conditions sometimes found in vulcanized rubber.

\section{ELECTRICAL ENGINEERING IN THE UNITED STATES}

ARCHER E. KNOWLTON, associate editor of the Electrical World (United States), in a review (J. Inst. Elec. Eng., 89, Pt. 1, No. 18, June, 1942) of electrical engineering progress in the United States during the past 2-21 years, points out that in 1941 and after, investigation and research have been redirected substantially towards military measures. Many developments arising out of the pressure for superior arms of every description are already regarded as having great import for the resumption of peace-time pursuits. Weights of electrical equipment are likely to be reduced as the results of iron research, and working temperatures of many kinds of equipment may be increased on account of alloy research.

Outstanding in the latter half of the period under review were the incentives to standardize, to simplify, to eke out and to compensate, in order that suddenly taxed systems could meet the exacting requirements of defence loadings with the least drain on capital, production facilities and installation forces. Also, the technique of proofing overhead lines against lightning made it possible to render a single line practically as reliable in performance as double circuits had been. Higher speed circuit-breakers, reclosure, earth-fault neutralizers, protector tubes, counterpoises, arresters, insulation co-ordination for impulses were all factors in creating effective immunity to the surges which precipitate outages.

In the field of power distribution, reliability of service vied with the imperative need for conservation of capital, and these two somewhat contradictory objectives can claim the bulk of the developments in power distribution. Among these might be mentioned sectionalization of radial feeders, reclosure, networks, banking of secondaries, rebalanced transformer losses, extensive application of corrective capacitors, surveys of thermal margins of apparatus, unit substations, step regulators, improved cables, etc. Change and uncertainty of location of defence loads, as well as the provision of spares for emergencies, have more recently prompted a considerable adoption of portable substations and capacitor banks.

The paper reviews more particularly and in a very condensed form the developments which have taken place in large generators, power generation, transformers, transmission, circuit interruption, relaying, lightning, switching and substations, cables, insulation, capacitors, power distribution, instruments, electronics and electrostatics, motors and control, industrial applications, transportations, rectifiers, electrothermics and electrochemistry, welding, illumination, and certain miscellaneous developments. 